\title{
The Value of Interdisciplinary Pain Management in Complex Regional Pain Syndrome Type I: A Prospective Outcome Study
}

\author{
Gurwant Singh, MD, Seth N. Willen, BS, Mark V. Boswell, MD, PhD, Jeffrey W. Janata, PhD, and
}

Thomas C. Chelimsky, MD

Background: Complex regional pain syndrome (CRPS) type I is a symptom complex of severe, chronic limb pain, often associated with allodynia, vasomotor, and sudomotor changes. Optimal management of this condition is not well understood. The role of a traditional, comprehensive pain management program with long-term follow-up has not been evaluated.

Objective: To define the benefit of the interdisciplinary approach in patients with CRPS type I.

Design: Prospective, case series, outcomes evaluation.

Methods: Patients with a diagnosis of CRPS type I entering the University Pain Center's intensive, outpatient pain management

Complex regional pain syndrome (CRPS) type I is a symptom complex of severe chronic limb pain, often associated with allodynia (pain induced by nonnoxious stimuli), vasomotor and sudomotor changes. Optimal CRPS management, particularly the value of an interdisciplinary program, is not well understood. Much work has defined specific aspects of the syndrome, especially in regard to physiology, diagnosis, and the effect of specific pharmacologic agents. However, little data exist to support a commonly recommended treatment approach, some form of rehabilitation or chronic pain management. This likely results from the vague syndrome definition, and the consequent inability to capture a uniform

From Departments of Neurology, Anesthesiology, and Psychiatry, University Hospitals of Cleveland, and Case Western Reserve University, Cleveland, Ohio. Address Correspondence: Thomas C. Chelimsky MD, Department of Neurology, University Hospitals of Cleveland, 11100 Euclid Avenue, Cleveland, $\mathrm{OH} 44106$.

E-mail: tcc3@case.edu

Support:There was no external funding in prepara-

tion of this manuscript

Conflict of Interest: None program were enrolled in an objective assessment study through the duration of the program, with a follow-up of 2 years. This program involved 4 weeks of interdisciplinary management comprised of 20 sessions of physical therapy, 20 sessions of occupational therapy, 12 sessions of water therapy, 20 sessions of group psychotherapy, stellate ganglion blocks, and drug therapy.

Outcome Measures: Specific objective measurements of upper extremity function, sensation and strength over time, and functional status 2 years after program completion.

Results: Upper extremity weight tolerance increased dramatically by 29 -pounds $(p<0.05)$. Function improved, with a 35 inch- pound gain in BTE (Baltimore Therapeutic Equipment) extension $(p<0.005)$ and a 50 inch-pound increase in flexion $(p<0.02)$. Jebsen-Taylor multifunctional testing (fine and gross motor skills) normalized from 72 to 48 seconds $(p<0.04)$. Stable anxiety levels despite increased patient effort implied improved pain tolerance. At the 2-year follow up, $75 \%$ of the patients were employed.

Conclusion: Patients with CRPS type I may benefit from a 4-week outpatient pain management program emphasizing rehabilitation.

Keywords: Complex regional pain syndrome, CRPS, reflex sympathetic dystrophy, RSD, interdisciplinary pain management, pain rehabilitation population. Only 3 studies (1-3) prospectively assessed the role of physical medicine, and only a single one in the context of a pain management program. Oerlemans et al (4) found that physical therapy (PT) and to a lesser extent occupational therapy (OT) produced significant and rapid improvement in limb function.

Does a traditional comprehensive pain management program truly provide long-term benefit to patients with CRPS I? This constitutes a difficult question, because of the complexity and non-uniformity of both the disorder (as presently defined), and the treatment. A simple initial approach that does not require uniformity of the population is to follow physiological changes over time in patients who are being treated in the best available way, using the comprehensive interdisciplinary approach.

This observational study evaluated 12 sequential patients with upper extremity CRPS entering the University Pain Center's interdisciplinary pain management program, with objective assessment and a follow-up of 2 years.

\section{Methods}

Subjects

During the 6 months of the enrollment period, 12 patients met definitive CRPS I upper extremity criteria and all agreed to participate. Criteria for inclusion were, age more than 18 years, diagnosis of CRPS I of the upper extremity as evidenced by at least three out of four of the following criteria for more than 30 days: 1) Pain out of proportion to known pathology, 2) Allodynia (pain induced by a non-noxious stimulus), 3) Vasomotor changes in the limb by examination (color, volume or sweat asymmetry), 4) Trophic changes (involving hair, nail, bone or skin). Exclusion criteria were: (1) inadequately treated major depression or anxiety (as assessed by the Pain Center psychologist); (2) incompletely diagnosed or not fully addressed medical illness, such as new angina or new onset diabetes (chronic illness with well-defined medical regimens were acceptable); (3) Pain too mild (VAS <4); (4) chemical dependency to an opioid or tranquilizing medication with 
inappropriate usage. We also enrolled 12 normal subjects to confirm norms for the various measures and to study the effect of repeated testing. Exclusion criteria for normal subjects were (1) pain or injury to upper extremity; (2) prescription medication with potential effect on peripheral or central autonomic functions. Patients were given written and oral information about the study.

\section{Informed Consent}

Written consent was obtained using an Institutional Review Board approved protocol and consent form.

\section{Study Design}

The goal of this prospective study was to determine if functional measurements made during a pain program could predict the long-term response of patients with CRPS I (and become useful to modify treatment course early for a particular patient). All patients underwent the 4week standard outpatient treatment program, with 4 weeks of interdisciplinary management by neurology, anesthesiology, psychology, and physical (PT) and occupational therapy (OT).

The treatment involved 20 sessions of PT, 20 sessions of OT, 12 sessions of water therapy and 20 sessions of group psychotherapy, with stellate ganglion blocks (when appropriate) administered 2/week during the first 2 weeks and 1/ week during the last 2 weeks, if one of the first two was effective. Care was taken not to make any measurements within 48 hours of blocks so as to assess overall progress and not momentary gains. Pharmacological management occurred without constraint, using the normal standard of practice, which included various analgesics and corticosteroids, as reported. We compared the functions of the affected and unaffected limbs during the program by repeating identical weekly measures. Since we were concerned about the learning effect derived from repeating the same test multiple times, we further compared the effect of test repetitions in the affected limb to the same effect in the unaffected limb and in the limbs of normal subjects. To reduce the testing variability, the same staff member always performed the same test on all subjects. Follow up to evaluate functional outcome was done by telephone questionnaire 2 years after program completion.

\section{Specific Tests}

There were 3 tests of function, 3 tests of physical capacity, 5 tests of psychological/behavioral attributes, 3 tests of autonomic function and 2 tests of sensation.

\section{Tests of Function}

1) Jebsen-Taylor Test (5): This widely used standardized test evaluates disability in patients with injuries and other conditions that affect hand function. Its seven sub-tests require timed performance of common daily tasks, with standardized verbal instructions as well as patient and item positions, and well-established norms: (1) Writing (12.2 \pm 3.5 sec.), (2) Turning over $3 \times 5$ cards (4.0 \pm 0.9 seconds), (3) Picking up small common objects, (paper clips, bottle caps) (5.9 \pm 1.0 seconds), (4) Simulated feeding (using spoon and kidney beans) $6.4 \pm$ 0.9 seconds), (5)- Stacking checkers (3.3 \pm 0.7 seconds), (6) Picking up large light objects (empty cans) (3.0 \pm 0.4 seconds), (7) Picking up large heavy objects ( $1 \mathrm{lb}$. coffee can) $(3.0 \pm 0.5$ seconds $)$. We confirmed the published norms with our own normal subjects. Our sub-test values for dominant and nondominant hands respectively follow: writing $(12 \pm 3.8,24 \pm 5.4$ secs. $)$, turning over $3 \times 5$ cards $(3.0 \pm 0.1,3.3 \pm$ 0.2 secs.), picking up small common objects $(5.5 \pm 0.9,5.9 \pm 0.4$ secs. $)$, simulated feeding $6.5 \pm 0.8,8.1 \pm 1.7$ secs.), stacking checkers $(3.0 \pm 0.1,3.7 \pm 1.2$ secs. $)$, picking up large light objects $(2.7 \pm 0.3,2.8 \pm$ 0.1 secs.) and picking up large heavy objects $(2.8 \pm 0.1,3.1 \pm 0.3$ secs. $)$.

2. Weight Bearing: Upper extremity weight bearing (weight and duration) was measured with a standard calibrated weight scale and a timer. The patient was in a quadruped position. Hand position was in slight extension with a towel roll interposed between the palmar surface of the hand and the scale. We determined the level of weight bearing tolerable for greater than $10 \mathrm{sec}$., but less than 1 minute, and recorded the average duration of 3 trials. Once the patient could exceed $3 \mathrm{~min}$. at that weight, a new, higher weight was used, determined in the same manner as above.

3. Pressure Tolerance: A highly sensitive and easily accessible area of skin was selected and marked for repeated measures, as was its symmetric counterpart on the uninvolved extremity. With the patient seated and the arm at a comfortable resting position, a pressure gauge identified a pressure (in ounces or pounds/ square inch) which the patient could tolerated between 10 and 60 seconds, and the exact duration of this tolerance was recorded. Subsequent duration measurements were done at the same level of pressure until the duration exceeded $3 \mathrm{~min}$ utes, when a new level of pressure bearable between 10 and 60 seconds was established.

\section{Tests of Physical Activity}

1. Baltimore Therapeutic Work Simulator Test (6): the Baltimore Therapeutic Work Simulator (BTE, Baltimore Therapeutic Equipment Company) quantified maximum isometric force and endurance. A variable resistance head accommodates a variety of tools designed to simulate common upper extremity work tasks. A force gauge measures effort. Tool \#171 was employed for wrist extension and flexion. A computer record stores test results. The BTE Company publishes standardized Procedures for testing along with norms in the BTE Clinical Application Manual. We confirmed the norms with our normal controls. The device performs 2 types of measurements: (1) maximum isometric force, the force against a static resistance, recorded in inch-pounds; (2) maximum dynamic endurance, the work produced to perform a specific muscle activity or joint movement, quantified in pounds-degrees of rotation. Data from both types of testing allowed comparison between the involved and uninvolved upper extremities in patients, dominant and non-dominant upper extremities in normals, agonist and antagonist muscle groups, individual performance and normative data, and individual performance and known requirements for particular job demands. In addition, performance consistency (standard error $<10 \%$ ) provided an assessment of subject effort in performing the test.

2. Goniometry: The goniometer provides a simple, validated and accurate measure of the range of motion across a joint. It measures active range when the subject moves the joint, and passive range if the examiner moves the joint. We measured both active and passive range across the wrist (flexion and extension), elbow (flexion and extension), shoulder (abduction and adduction) and any involved digits (metacarpophalangeal, proximal and distal interphalangeal joints). 
3. Volume: Limb volume, measured with a volumeter, quantified the difference in involved and uninvolved upper extremity limb volume in milliliters, providing a measure of swelling and atrophy.

Tests of Psychological/Behavioral Attributes

To measure pain intensity, the visual analog scale was completed weekly, when the other measurements were taken. We also used the McGill Pain Questionnaire $(7,8)$, a widely used, standardized quantitative index of pain. Patients were asked to describe their pain by endorsing descriptive adjectives that fall into a series of subclasses, which yields three measures of subjective pain intensity. The CES-D (9), a brief measure of depression designed particularly for use in medical populations, was used to assess depression. Unlike other methods used to quantify depressive symptoms, the CES-D loads very little on the physical symptoms of depression, and as such, is ideally suited for identifying depression in a chronic pain population. We also administered the Pain Locus of Control (PLOC) (10), a brief scale used to assess internal ("I'm responsible for my pain") vs. external ("You're the doctor, please fix me") locus of control specific to pain, the Pain Cognitions Questionnaire (PCQ) and Pain Situations Questionnaire (PSQ) (11), scales measuring fear of pain and predicting the severity of avoidance of use of a painful limb. The PCQ consists of eighteen items scored on a 5-point scale and the PSQ consists of 20 items scored on an 8-point scale.

\section{Autonomic Testing}

(see references for more detailed methods description)

1. Point temperatures (12): An infrared thermometer measured infrared emittance from the skin in real-time (about $5 \mathrm{sec} /$ point). There was no physical contact between the skin and the instrument.

2. Resting sweat output (12): Dry nitrogen gas passes through a $1 \mathrm{~cm}$ diameter capsule made of lexan applied to the skin, carrying the added moisture from sweat to a thermal conductivity cell. This instrument provides a dynamic, quantitative readout of the sweat output.

3. Muscle blood flow: A blood pressure cuff inflated to above arterial systolic pressure, at the level of the wrist, isolated forearm circulation from the hand.
A second blood pressure cuff, just above the elbow, was rapidly inflated to just below diastolic pressure (usually $50 \mathrm{mmHg}$ ) for 6 seconds. The slope of expansion of the forearm measured over these $6 \mathrm{sec}$ onds by a mercury-filled strain gauge provides an accurate index of muscle blood flow. Both cuffs were then immediately deflated.

\section{Statistical analysis}

Affected to unaffected limb comparisons were performed in individual subjects for each measure, comparing dynamic progress in the unaffected limb (presumed to be a learning and strengthening effect only) to that in the affected limb (presumed to be a combination of learning, strengthening and physiologic improvement). We utilized both the paired t-test between limbs, and trend analysis across multiple measures in time. We also compared the unaffected limb in patients to the change in normal subjects. In an attempt to find predictors of success, subsequent evaluation of the entire group utilized a series of analyses of covariance with repeated measures. To equate patients at baseline, the baseline values of each dependent measure were included as the co-variate. The large quantity of comparisons required an adjustment in the level of statistical significance with a Bonferroni correction.

\section{Results}

The demographic features of the study group are shown in table 1 . The mean age was 40 years (range: $26-67$ years), $75 \%$ were female patients, and $58 \%$ had right upper extremity CRPS. Preceding trauma occurred in $92 \%$. The mean

Table 1. Demographic data for the 12 subjects in the study
\begin{tabular}{|c|c|c|c|c|c|}
\hline $\begin{array}{c}\text { Patient } \\
\text { Number }\end{array}$ & Age & Sex & $\begin{array}{c}\text { Side } \\
\text { involved }\end{array}$ & $\begin{array}{c}\text { Symptom Duration } \\
\text { (months) }\end{array}$ & $\begin{array}{c}\text { Preceding } \\
\text { trauma }\end{array}$ \\
\hline $\mathbf{1}$ & 35 & F & Right & 18 & Yes \\
\hline $\mathbf{2}$ & 45 & F & Right & 14 & Yes \\
\hline $\mathbf{3}$ & 40 & F & Right & 28 & Yes \\
\hline $\mathbf{4}$ & 30 & F & Right & 25 & Yes \\
\hline $\mathbf{5}$ & 45 & F & Left & 12 & Yes \\
\hline $\mathbf{6}$ & 26 & M & Right & 6 & Yes \\
\hline $\mathbf{7}$ & 40 & M & Right & 7 & Yes \\
\hline $\mathbf{8}$ & 28 & F & Right & 13 & Yes \\
\hline $\mathbf{9}$ & 46 & M & Left & 19 & Yes \\
\hline $\mathbf{1 0}$ & 39 & F & Left & 7 & No \\
\hline $\mathbf{1 1}$ & 67 & F & Left & 6 & Yes \\
\hline $\mathbf{1 2}$ & 43 & F & Left & 101 & Yes \\
\hline
\end{tabular}

$\overline{\text { Table 2. Effect of stellate ganglion blocks in patients who received them during }}$ the program

\begin{tabular}{|c|c|c|c|c|c|}
\hline $\begin{array}{c}\text { Patient } \\
\text { Number }\end{array}$ & $\begin{array}{c}\text { Total Number } \\
\text { of Blocks }\end{array}$ & $\begin{array}{c}\text { Block } \\
\text { Number }\end{array}$ & $\begin{array}{c}\text { Program Day } \\
\text { Received }\end{array}$ & $\begin{array}{c}\text { Relief } \\
\text { (\%) }\end{array}$ & $\begin{array}{c}\text { Duration of } \\
\text { Pain Relief }\end{array}$ \\
\hline 5 & 2 & 1. & Day \# 4 & 60 & 7 days \\
\hline 6 & 1 & 2. & Day \# 11 & 100 & 21 days \\
\hline 8 & 4 & 1. & Day \# 4 & 100 & 3 days \\
\hline & & 1. & Day \# 4 & 70 & 8 hours \\
\hline & 2. & Day \# 8 & 50 & 3 days \\
\hline 10 & 3. & Day \# 11 & 100 & 2 days \\
\hline & & 1 & Day \# 15 & 100 & 6 days \\
\hline & & 2 & Day \# 4 & 80 & 4 days \\
\hline & & 3 & Day \# 11 & 95 & 3 days \\
\hline
\end{tabular}




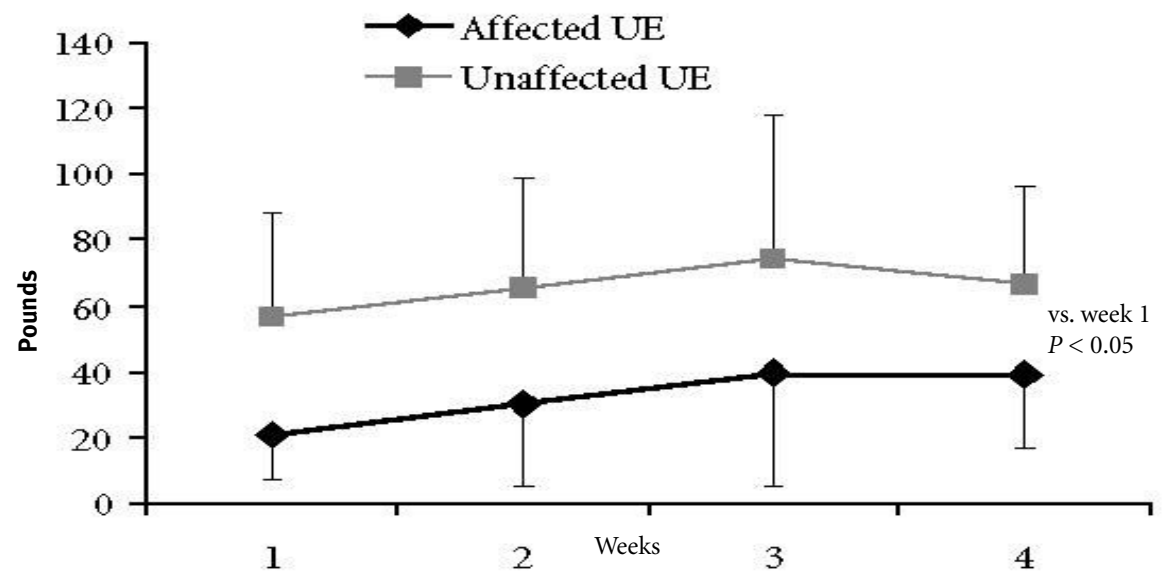

Fig 1. Weekly change in weight bearing measured over the 4-week program, comparing affected with unaffected upper extremities. The statistically significant difference from week 1 to week 4 occurred only in the affected extremity.

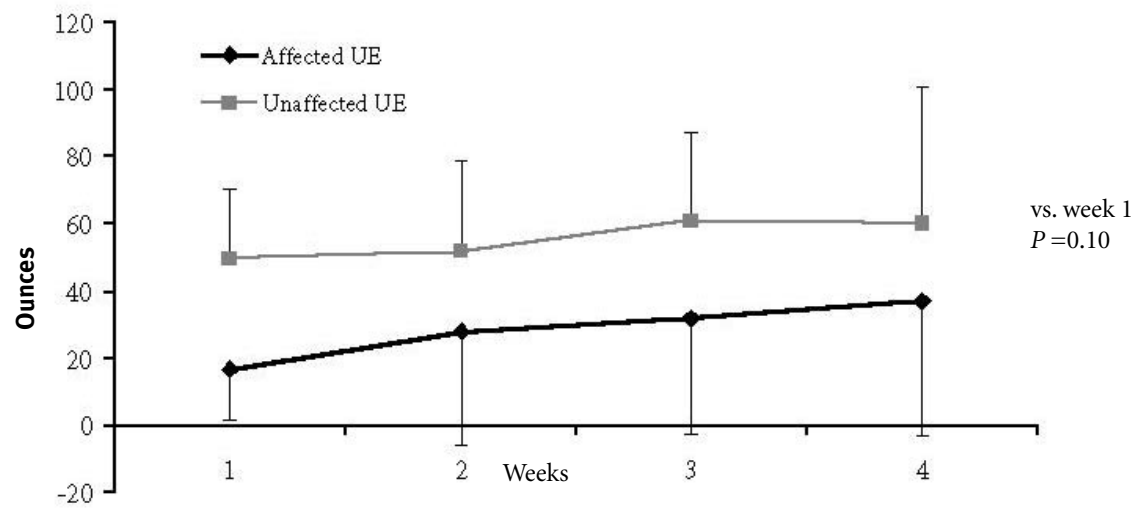

Fig 2. Weekly change in pressure tolerance measured over the 4-week program, comparing affected with unaffected upper extremities. Only a trend towards improved pressure tolerance occurred in the affected extremity.

duration of symptoms from the point of injury to participation in the pain management program was 18 months, ranging from 6-28 months, with a single patient at 101 months. Stellate ganglion blocks were offered to patients, though most declined (table 2). As shown, blocks generally produced partial or complete pain relief lasting from 8 hours to 3 or more days.

Statistically significant differences occurred at the end of 4 weeks in all the outcome measures except limb volume. The bearing of weight almost dou- bled (Fig. 1) at the end of four weeks, as did pressure tolerance (Fig. 2). Force and endurance as tested with the BTE also showed a significant improvement (Fig. 3). A profound improvement in hand function was reflected by the JT (Fig. 4). Limb volume showed a trend towards reduction. Other physiologic data obtained through autonomic testing were inconsistent. Repeated autonomic testing did not correlate in any way with clinical improvement.

Behavioral assessment showed no significant change in pain tolerance as measured by the different pain questionnaires. However, anxiety levels remained stable, despite intensive PT and OT participation, suggesting an overall improvement in functioning.

At the 2 -year assessment, $75 \%$ of the patients were employed, the majority of them in a job different from the one they had left after the onset of CRPS. Of those interviewed, $66 \%$ perceived the program as significantly helpful, with particular credit attributed to self-pacing strategies 


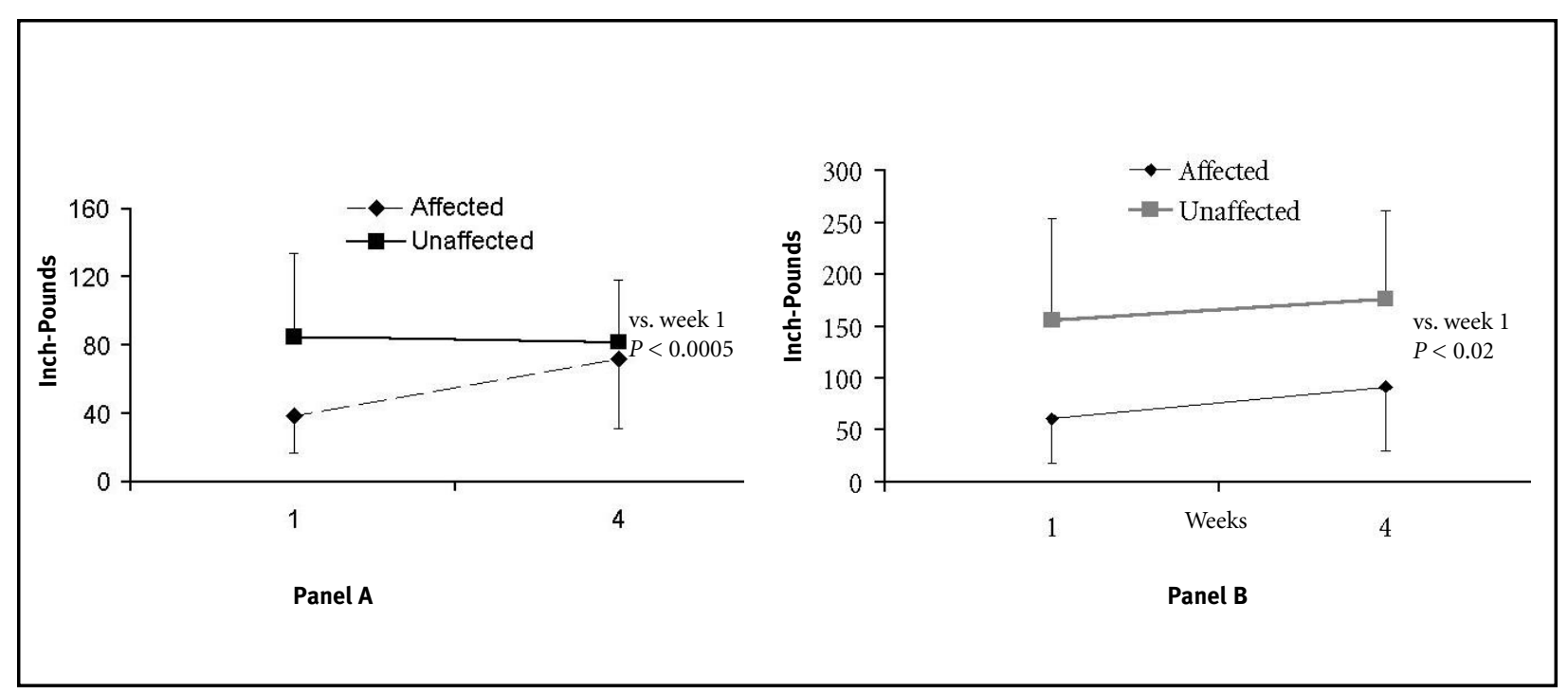

Fig 3. Upper extremity isometric endurance, as measured by the BTE, comparing affected with unaffected upper extremities Panel A: Wrist extensors, Panel B: Wrist flexors. Although both extensors and flexors were affected, the most dramatic improvement occurred in the extensors, which normalized.

and use of correct body mechanics. They were more active and optimistic about long-term outcome, despite increased or more widely distributed pain related to CRPS in $50 \%$ and a similar proportion still using opioids (Table 3 ).

\section{DISCUSSION}

To our knowledge, this is the first study to define longitudinal objective changes in patients with CRPS undergoing comprehensive, outpatient pain management in a 4-week interdisciplin- ary program. This study indicates that an aggressive program of this type produces both subjective and objective improvement in function. Improvement was not just short-term, but appeared to have a persistent effect. The program was perceived to be helpful by a majority of pa-

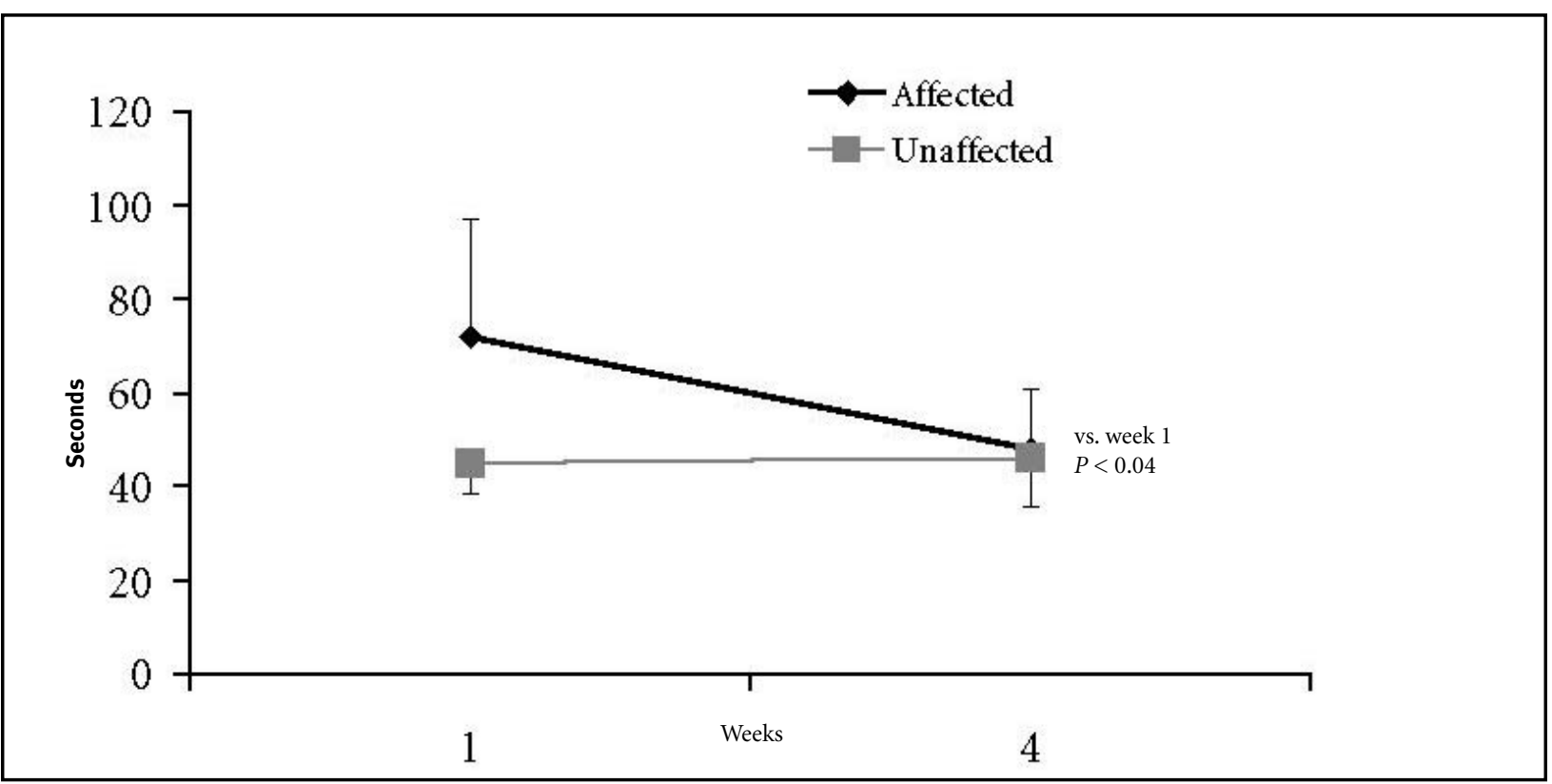

Fig 4. Improvement in fine and gross motor skills as measured by the Jebsen Taylor test, comparing affected with unaffected limb (see methods for the 7 subsets of this procedure). The y-axis is measured in seconds, the total time for all tests combined. The affected extremity began at nearly double the time, and had normalized by the end of the program. Interestingly the unaffected extremity did not change, suggesting very little learning effect. 
Table 3. Two-year program outcome, based on a telephone interview

\begin{tabular}{|c|c|c|c|c|c|}
\hline Patient Number & Employed? & Degree of pain & Spread of pain & Pain program helpful & Using Opioids \\
\hline 1 & Yes & Same & Yes & Extremely helpful & Yes \\
\hline 2 & Yes & Same & No & Extremely helpful & Yes \\
\hline 3 & Yes & Less & No & Extremely helpful & Yes \\
\hline 4 & Unable to & contact & & & \\
\hline 5 & Yes & Less & Yes & Very helpful & No \\
\hline 6 & Yes (in school) & Unable to & obtain & information & \\
\hline 7 & No & Same & Yes & Somewhat helpful & Yes \\
\hline 8 & Yes & Worse & Yes & Extremely helpful & Yes \\
\hline 9 & Yes & Same & No & helpful & No \\
\hline 10 & Yes & Worse & No & Neutral & Yes \\
\hline 11 & Neither before nor now & Less & No & Yes & No \\
\hline 12 & Yes & Less & No & Neutral & No \\
\hline
\end{tabular}

tients 2 years later. Most patients were still employed (Table 2). Standard measures of the impact of PT and OT showed significant improvement. It is therefore likely that PT and OT were major contributors to the patient's progress. However, psychological training is probably critical to redirect patients away from a pain focus towards function, which allows the rehabilitation to occur. To wit, many patients had previously not benefited from excellent PT alone. The absence of any significant decrease in pain level while physical gains increased suggests that focusing on improved function rather than pain reduction is a viable approach, since most patients $(75 \%)$ returned to work.

The psychological indices were a little disappointing in not demonstrating more clear-cut changes, such as a shift from external to internal locus of control, or a clear reduction in anxiety to pain, fear or pain, or depression scales. However, we believe a relative improvement in anxiety to pain must have occurred, because psychosocial testing showed stable anxiety levels despite increased patient effort and progressively higher expectations. Anxiety related to pain usually correlates with effort, therefore stable anxiety levels in the face of increased physical challenge likely represent improved pain tolerance and a relative anxiety decrease.

Efforts to minimize pain and improve patient comfort occurred through blocks and analgesic medications. Although stellate ganglion blocks exert a transient pain-relieving effect (hours to days) in the majority of patients, they could produce long-term benefit through increased patient function in physical and occupational therapy. We could not directly demonstrate such a relationship, due to lack of statistical power.

Conventional adjuvant analgesics (tricyclic antidepressants, anticonvulsants and non-steroidal anti-inflammatory agents), oral corticosteroids (prednisone) and where appropriate, opioids, were tried and continued when helpful. Although the pharmacological strategy was identical across patients, the drug selection (based on effectiveness and adverse effects) was clearly different from patient to patient, and could be viewed as a confounding variable. However, the uniformity of our findings across this population becomes even more striking in this context, and further suggests the rehabilitation program itself as the source of the patients' improvement. Indeed, approximately half the patients had been on the identical medications prior to program initiation, without much improvement.

Limb volume decreased in proportion to functional improvement as might be expected if soft tissue swelling dissipates with treatment. This was only a trend and did not reach statistical significance, perhaps because of the relatively later stage of the disease for most patients, when swelling is generally less dramatic than at the onset of the disorder. Autonomic testing showed no uniformly consistent changes as patients progressed through the program. In particular, sweat rates did not increase or decrease. This may reflect the variable state of disease at the time of patient presentation to the program.

Our goal was a detailed description of the objective changes in a small cohort of 12 patients undergoing a traditional intensive pain program. Untreated controls with CRPS were not used in this study to assess the effectiveness of the treatment because: 1) this was a pilot study, 2) this is the currently accepted method of treatment, 3) based on historical controls and patient reports prior to program entry, we believe the disease progresses without such management. This report provides a set of methods to study larger populations with carefully designed control groups. An untreated control group could be constructed either by delaying treatment for a specific time interval and comparing progress during this time, or withholding specific treatment components to assess their impact.

Other studies also have evaluated the benefits of rehabilitation in CRPS but none have included long-term life-based functional outcomes such as return to work. Davidoff (2) quantified pain levels using PRI-T (Pain Rating Index), VAS (Visual Analogue Scale) and NWC (Number of Words Chosen) to measure correlation between pain and clinical improvement in 17 CRPS patients participating in an exercise program. The pain levels as measured by VAS correlated strongly with the limb volume. The PRI-T and NWC correlated only with AROM (active range of motion) of lower extremities and joint pain indices (not used in our study). The range of motion improved significantly in our patients as well. The methods employed in that study guided the present protocol.

Two prospective studies by Oerlemans $(4,13)$ reported the benefit of physical and occupational therapy in 
CRPS patients. The first was a prospective evaluation of 135 CRPS patients in a randomized controlled trial, with 1-year follow-up. The primary outcome measured was the change in an impairment level sumscore (ISS) (4). PT and to a lesser extent OT resulted in a significant improvement of the ISS as compared to the controls. Improvement in ISS using intention to treat analysis was $53 \%$ for PT and $48 \%$ for OT. In a previous study the same author compared outcome in CRPS patients treated with $\mathrm{PT}$ and OT in a prospective trial and found PT and OT to be equally effective. The weakness of these studies lies in the ISS measurement, which only reflects symptomatic improvement, rather than externally measured "true-life" function such as return to work. In addition, none of these publications addressed the complex neuro-psychological aspects of treatment.

In summary, this study found that an intensive approach to upper extremity CRPS, combining physical and occupational therapy under-girded by an aggressive neuropsychological behavioral strategy, and aided by interventional and medical treatment, produced significant, persistent improvement in function . The participating subjects were diverse in age, race and disease chronicity, suggesting that our findings may be generalizable across a broader population. This management strategy may improve the longterm prognosis in such patients to a status better than previously believed. Rigorously controlled studies will provide a clearer answer to this question.

\section{CONCLUSION}

This limited prospective evaluation showed that patients with CRPS type I may benefit from a 4-week outpatient pain management program emphasizing rehabilitation. The surprising finding of long-term progress in most patients prevented identification of outcome predictors.

\section{ACKNOWLEDGMENTS}

The Staff of the University Pain Center provided invaluable assistance with this study.

\section{Author Affiliation: \\ Gurwant Singh, MD \\ Pain Medicine Fellow \\ Department of Neurology \\ University Hospitals of Cleveland \\ and Case Western Reserve University \\ 11100 Euclid Avenue \\ Cleveland, OH 44106}

Seth N. Willen, BS

Medical Student

Case Western Reserve University

School of Medicine

10900 Euclid Avenue

Cleveland, $\mathrm{OH} 44106$

\section{Mark V. Boswell, MD, PhD}

Associate Professor of Anesthesiology

Chief, Division of Pain Medicine

University Hospitals of Cleveland

and Case Western Reserve University

11100 Euclid Avenue

Cleveland, OH 44106

E-mail: mark.boswell@uhhs.com

\section{Jeffrey W. Janata, PhD}

Assistant Professor of Psychiatry

Director, Behavioral Medicine

Program

University Hospitals of Cleveland

Case Western Reserve University

11100 Euclid Avenue

Cleveland, $\mathrm{OH} 44106$

Email: jeffrey.Janata@uhhs.com

Thomas C. Chelimsky, MD

Associate Professor of Neurology

University Hospitals of Cleveland

and Case Western Reserve University

11100 Euclid Avenue

Cleveland, OH 44106

Email: tcc3@case.edu

\section{REFERENCES}

1. Watson HK, Carlson L. Treatment of reflex sympathetic dystrophy of the hand with an active "stress loading" program. J Hand Surgery 1987; 12A:779-785

2. Davidoff G, Morey K, Amann M et al. Pain measurement in reflex sympathetic dystrophy syndrome. Pain 1988; 32:27-34.

3. Oerlemans HM, Goris JA, de Boo T et al. Do physical therapy and occupational therapy reduce the impairment percentage in reflex sympathetic dystrophy? Am J Phys Med Rehabil 1999; 78:533-539

4. Oerlemans HM, Oostendorp RA, de Boo T et al. Adjuvant physical therapy versus occupational therapy in patients with reflex sympathetic dystrophy/complex regional pain syndrome type I. Arch Phys Med Rehabil. 2000; 81:49-56.

5. Jebsen R, Taylor N. An objective and standardized test of hand function. Arch Phys Med Rehabil 1969; 50:311-319.

6. Baltimore Therapeutic Company: BTE Work Simulator Clinical Applications. Baltimore, 1992.

7. Melzack R. The McGill Pain Questionnaire: major properties and scoring methods. Pain 1975; 1:277-299.

8. Turk D, Melzack R. Handbook of pain assessment. Guilford Press, New York, 1992.

9. Radloff L. The CES-D scale: A self-report depression scale for research in the general population. Applied Psychological Measurement 1977; 1:385-401

10. Penzien DB: The pain locus of control scale. In Proceedings of the Eighth Annual Scientific Meeting of the American Pain Society. 1989; 68-72

11. Suttenfield C. Fear of pain: A cognitive model for the development and maintenance of chronic pain behavior. Doctoral Dissertation. George Mason University Fairfax, VA, 1995.

12. Chelimsky T, Low P, Naessens J et al. Value of autonomic testing in reflex sympathetic dystrophy. Mayo Clinic Proceedings 1995; 70:1029-1040.

13. Oerlemans HM, Oostendorp RA, de Boo T et al. Pain and reduced mobility in complex regional pain syndrome l: Outcome of a prospective randomized controlled clinical trial of adjuvant physical therapy versus occupational therapy. Pain 1999; 83: 77-83. 
